Hardy-Ramanujan Journal

Vol.11 (1988) 30-39

\title{
ON $n$ NUMBERS ON A CIRCLE
}

\author{
M. Misiurewicz and A. Schinzel
}

The following problem has been considered by B. Freeman [2], M. Lotan [3], L. Carlitz and R. Scoville [1].

A positive integer $n$ is fixed. There is given a vector

$$
a=a(0)=\left[a_{1}(0), \ldots, a_{n}(0)\right] \in \mathbf{R}^{n}
$$

and an infinite sequence

$$
a(t)=\left[a_{1}(t), \ldots, a_{n}(t)\right]
$$

is formed by means of the formulae

$$
a_{i}(t+1)=\left|a_{i}(t)-a_{i+1}(t)\right|
$$

where the addition of indices is $\bmod n$. A convenient method to think about the numbers $a_{i}(t)$ is to place them on the unit circle, at the $n$-th roots of 1 . The problem is to describe the behaviour of the sequence $a(t)$ at infinity.

If $\mathrm{a} \in \mathrm{Z}^{n}$ and $n=2^{k}$ then we have $\mathrm{a}(t)=0$ for all large $t$. This was proved in [2]. If $a \in \mathbf{Z}^{n}$ and $n \neq 2^{k}$ then there exists an integer $c$ such that $a(t) \in\{0, c\}^{n}$ for all large $t$. This has been deduced in [1] from a statement equivalent to Lemma 1 below, however the proof of this statement has been in our opinion insufficient. If $a \in \mathbf{R}^{n}$ then it may happen that $\lim _{t \rightarrow \infty} \mathbf{a}(t)=0$, but $a(t) \neq 0$ for all $t$. This was shown in [1] by an example and for $n=4$ all such cases have been determined in [3].

O. Radic has suggested (unpublished) that if $a \in \mathbf{R}^{n}$ and $n=2^{k}$ then $\lim _{t \rightarrow \infty} \mathbf{a}(t)=0$. This will be proved below; moreover all possible limit points of 
the sequence $a(t)$ will be determined for every $n$. The problem that remains open is the following.

Is it true that for every $n$ and every $\mathbf{a} \in \mathbf{R}^{n}$ either $\lim _{t \rightarrow \infty} \mathbf{a}(t)=0$ or there exists $c \in \mathbf{R}$ such that $\mathbf{a}(t) \in\{0, c\}^{n}$ for all sufficiently large $t$ ?

We can look at the whole situation in the following way. There is a continuous map $T: \mathbf{R}^{n} \rightarrow \mathbf{R}^{n}$ given by a formula

$$
T\left(\left[b_{1}, \ldots, b_{n}\right]\right)=\left[\left|b_{1}-b_{2}\right|, \ldots,\left|b_{n-1}-b_{n}\right|,\left|b_{n}-b_{1}\right|\right] .
$$

Then $\mathbf{a}(t)=T^{t}(\mathbf{a})$, where $T^{t}$ denotes the $t$-th iterate of $T$. The set of all limit points of the sequence $a(t)$ (i.e. of the trajectory of $a$ ) is the $\omega$-limit set of the point a (see e.g. [4]).

Clearly, $T\left(\mathbf{R}^{n}\right) \subset \mathbf{R}_{+}^{n}$, so we may restrict our attention to $\mathbf{R}_{+}^{n}$. For a vector $\mathbf{b}=\left[b_{1}, \ldots, b_{n}\right]$ we set $|\mathbf{b}|=\max _{1 \leq i \leq n}\left|b_{i}\right|$. Notice that

$$
|T(\mathbf{b})| \leq|\mathbf{b}| \quad \text { for all } \mathbf{b} \in \mathbf{R}_{+}^{n} \text {. }
$$

This implies in particular that for each $\mathbf{a} \in \mathbf{R}_{+}^{\mathbf{n}}$ the whole sequence $\mathbf{a}(t)$ is contained in the compact set

$$
\left\{\mathbf{b} \in \mathbf{R}_{+}^{n}:|\mathbf{b}| \leq|\mathbf{a}|\right\} \text {. }
$$

Therefore this sequence has at least one limit point.

Using this approach we may state our main results in the following way. There is a finite set of periodic orbits such that every trajectory of $T$ is attracted to one of them; moreover we find all these periodic orbits.

More precisely, we shall prove:

THEOREM 1. For every vector $\mathbf{a} \in \mathbf{R}^{n}$ and a limit point $\mathbf{p}$ of the sequence $\mathrm{a}(t)$ we have

$$
\begin{gathered}
\mathbf{p}=c \mathbf{e} \text {, where } c=\lim _{t \rightarrow \infty}|\mathbf{a}(t)| \text { and } \mathbf{e}=\left[\varepsilon_{1}, \ldots, \varepsilon_{n}\right] \in\{0,1\}^{n} . \\
1+x^{2 \nu} \mid \sum_{i=1}^{n} \varepsilon_{i} x^{i-1}(\bmod 2), \text { where } 2^{\nu} \| n .
\end{gathered}
$$


COROLLARY. If $n=2^{2}$ then for all vectors $\mathbf{a} \in \mathbf{R}^{n}$ we bise

$$
\lim _{t \rightarrow \infty} \mathbf{a}(t)=0
$$

THEOREM 2. If a. $=c e$, where $c \in \mathbf{R}_{+}$and $\mathbf{e}=\left[\varepsilon_{1}, \ldots, \varepsilon_{\mathrm{n}}\right] \in\{0,1\}^{\mathbf{n}}$ satisfies (3) ther. $\mathbf{a}(N)=\mathbf{a}$ for some $N>0$, i.e. the orbit of $\mathbf{a}$ is periodic.

ine proof of Theorem 1 is based on three lemmata.

LEMMA 1. Assume that $\mathrm{p}=\left[p_{1}, \ldots, p_{n}\right] \in \mathbf{R}_{+}^{n}, 1 \leq k \leq n$ and

$$
0<p_{k}<|\mathbf{p}| \text {. }
$$

Then

$$
|\mathbf{p}(\boldsymbol{n}-1)|<|\mathbf{p}|
$$

PROOF. We claim that for every $i \geq 0$ with $|\mathbf{p}(i)|=|\mathbf{p}|$ there exist integers $j_{i}^{ \pm}$with the following properties:

$$
\begin{aligned}
j_{i}^{+} \geq 0, j_{i}^{-} \leq-i, \\
0 \leq p_{k+j}(i)<|\mathrm{p}| \text { for } j \in\left(j_{i}^{-}, j_{i}^{+}\right), \\
0<p_{k+j_{i}^{ \pm}}(i)<|\mathrm{p}| .
\end{aligned}
$$

We use the induction on $i$. For $i=0$ we take $j_{0}^{ \pm}=0$. Assume that the claim is true for a certain $i$. If $|\mathbf{p}(i+1)|=|\mathbf{p}|$ then also $|p(i)|=|\mathbf{p}|$ and we may assume without loss of generality that $j_{i}^{+}, j_{i}^{-}$are the greatest and the least integer respectively satisfying the conditions (4) - (6). (Note that $\left(j_{i}^{-}, j_{i}^{+}\right)=\left(j_{i}^{-}, 0\right] \cup\left[0, j_{i}^{+}\right), 80$ that the assumption is logically correct). Hence

$$
p_{k+j_{i}^{ \pm} \pm 1}(i) \in\{0,|p|\}
$$


We now take

$$
j_{i+1}^{+}=j_{i}^{+}, j_{i+1}^{-}=j_{i}^{-}-1
$$

It follows from (4) that

$$
j_{i+1}^{+} \geq 0, j_{i+1}^{-} \leq-i-1
$$

Moreover, if $j \in\left(j_{i+1}^{-}, j_{i+1}^{+}\right)=\left[j_{i}^{-}, j_{i}^{+}\right)$then we have by (5) and (6),

$$
p_{k+j}(i+1)=\left|p_{k+j+1}(i)-p_{k+j}(i)\right|<|p| \text {. }
$$

Finally, by (6) and (7),

$$
p_{k+j_{i+1}^{ \pm}}(i+1)=\left|p_{k+j_{i}^{ \pm} \pm 1}(i)-p_{k+j_{i}^{ \pm}}(i)\right| \in(0,|\mathbf{p}|)
$$

This completes the induction step and the proof of the claim.

Assume now that $|p(n-1)|=|p|$. By (4) - (6) for $i=n-1$, there exist integers $j_{n-1}^{+} \geq 0$ and $j_{n-1}^{-} \leq 1-n$ such that

$$
0 \leq p_{k+j}(n-1)<|p| \text { for all } j \in\left[j_{n-1}^{-}, j_{n-1}^{+}\right] \text {. }
$$

However, the interval $\left[j_{n-1}^{-}, j_{n-1}^{+}\right]$contains all residue classes $\bmod n$, and hence $|\mathbf{p}(n-1)|<|\mathbf{p}|$, contrary to the assumption.

LEMMA 2. If $\mathbf{p}=\left[p_{1}, \ldots, p_{n}\right]$ is a limit point of the sequence $\mathbf{a}(t)$ then

$$
\operatorname{Card}\left(\left\{p_{1}, \ldots, p_{n}\right\} \backslash\{0\}\right) \leq 1 .
$$

PROOF. We may assume that $\mathbf{a} \in \mathbf{R}_{+}^{n}$. Then, by (1), the sequence $|\mathbf{a}(t)|$ is non-increasing and therefore the limit

$$
c=\lim _{t \rightarrow \infty}|\mathbf{a}(t)|
$$


exists. Suppose that

$$
\operatorname{Card}\left(\left\{p_{1}, \ldots, p_{n}\right\} \backslash\{0\}\right) \geq 2
$$

and that

$$
\mathbf{p}=\lim _{k \rightarrow \infty} \mathbf{a}\left(t_{k}\right)
$$

for some sequence $t_{k} \rightarrow \infty$. Since $p \in \mathbf{R}_{+}^{n}$ and by (9), we can use Lemma 1 . We get, taking (8) into account,

$$
\left|T^{n-1}(\mathbf{p})\right|<|\mathbf{p}|=c
$$

The map $T$ is continuous and therefore from (10) we obtain

$$
\lim _{k \rightarrow \infty} a\left(t_{k+n-1}\right)=T^{m-1}(\mathbf{p})
$$

Together with (8) and (11) this gives a contradiction.

LEMMA 3. If $\mathrm{e}=\left[\varepsilon_{1}, \ldots, \varepsilon_{n}\right] \in \mathbf{Z}^{n}$ and $2^{\nu} \| n$ then

$$
1+x^{2^{n}} \mid \sum_{i=1}^{n} \varepsilon_{i}\left(2^{\nu}\right) x^{i-1}(\bmod 2) .
$$

PROOF. By the definition of $\mathbf{a}(t)$,

$$
\begin{gathered}
\sum_{i=1}^{n} \varepsilon_{i}(t+1) x^{i} \equiv \sum_{i=1}^{n}\left(\varepsilon_{i}(t)+\varepsilon_{i+1}(t)\right) x^{i} \equiv \\
\sum_{i=1}^{n} \varepsilon_{i}(t) x^{i}+\sum_{i=1}^{n} \varepsilon_{i}(t) x^{i-1}+\varepsilon_{1}(t) x^{n} \equiv \\
(1+x) \sum_{i=1}^{n} \varepsilon_{i}(t) x^{i-1}+a_{1}(t)\left(1+x^{n}\right) \bmod 2
\end{gathered}
$$


(cf. $[1$, p. 298]). Hence,

$$
x \sum_{i=1}^{n} \varepsilon_{i}(t+1) x^{i-1} \equiv(1+x) \sum_{i=1}^{n} \varepsilon_{i}(t) x^{i-1} \bmod \left(2,1+x^{2^{\nu}}\right),
$$

and by induction on $j$

$$
x^{j} \sum_{i=1}^{n} \varepsilon_{i}(j) x^{i-1} \equiv(1+x)^{j} \sum_{i=1}^{n} \varepsilon_{i} x^{i-1} \bmod \left(2,1+x^{2^{\nu}}\right),
$$

Taking $j=2^{\nu}$ and observing that

$$
(1+x)^{2^{\nu}} \equiv 1+x^{2^{\nu}} \bmod 2
$$

we obtain the lemma.

PROOF OF THEOREM 1. As we noticed already, the limit (8) exists. Now (2) follows immediately from Lemma 2 (if $c=0$ then we can take $\mathbf{e}=\mathbf{0}$ ).

Denote

$$
\begin{gathered}
P=\left\{c e: e=\left[\varepsilon_{1}, \ldots, \varepsilon_{n}\right] \in\{0,1\}^{n}\right\} \\
Q=\left\{c e: e=\left[\varepsilon_{1}, \ldots, \varepsilon_{n}\right] \in\{0,1\}^{n} \text { and (3) holds }\right\}
\end{gathered}
$$

From Lemma 3 and the fact that

$$
T(\mathrm{cb})=c T(\mathbf{b}) \text { for all } \mathbf{b} \text { in } \mathbf{R}^{n}
$$

we obtain

$$
T^{2^{\nu}}(P) \subset Q
$$

Since the whole sequence $a(t)$ lies in a compact set, we have by (2),

$$
\lim _{t \rightarrow \infty}\left(\min _{\mathbf{p} \in P}|\mathbf{a}(t)-\mathbf{p}|\right)=0 .
$$


By this, (14) and continuity of $T$,

$$
\lim _{t \rightarrow \infty}\left(\min _{\mathbf{p} \in Q}|\mathbf{a}(t)-\mathbf{p}|\right)=0
$$

Therefore all limit points of the sequence a(t) belong to $Q$.

PROOF OF COROLLARY. If $n=2^{\nu}$ then the only vector e $\in\{0,1\}^{n}$ satisfying (3) is $e=0$. Box

PROOF OF THEOREM 2. By (13) and since the set

$$
S=\left\{\mathbf{e}: \mathbf{e}=\left[\varepsilon_{1}, \ldots, \varepsilon_{n}\right] \in\{0,1\}^{n} \text { and (3) holds }\right\}
$$

is finite, it is enough to prove that the map $T$ maps $S$ bijectively onto itself.

By (12), $T(S) \subset S$. Therefore it remains to prove that if $e=\left[\varepsilon_{1}, \ldots, \varepsilon_{n}\right]$ and $\mathrm{d}=\left[\delta_{1}, \ldots, \delta_{n}\right]$ belong to $S$ and $\mathrm{e} \neq \mathrm{d}$ then $T(\mathrm{e}) \neq T(\mathrm{~d})$. Suppose that $T(\mathbf{e})=T(\mathbf{d})$. For each $i$ we have

$$
\left|\epsilon_{i}-\varepsilon_{i+1}\right|=\left|\delta_{i}-\delta_{i+1}\right|
$$

so $\varepsilon_{i}=\delta_{i}$ if and only if $\varepsilon_{i+1}=\delta_{i+1}$. Since $\neq \mathbf{d}$, for some $j$ we have $\varepsilon_{j} \neq \delta_{j}$. Then by induction we obtain $\varepsilon_{i} \neq \delta_{i}$ for all $i$. Since $\varepsilon_{i}, \delta_{i} \in\{0,1\}$, this means that $\varepsilon_{i}+\delta_{i}=1$ for all $i$. Therefore

$$
1+x^{2^{*}} \mid \sum_{i=1}^{n} x^{i-1}(\bmod 2)
$$

80

$$
\left(1+x^{2}\right)(1+x) \mid 1+x^{n}(\bmod 2) .
$$

Setting $m=n 2^{-\nu}$, we get

$$
1+x \mid \sum_{j=0}^{m-1} x^{j 2^{\nu}}(\bmod 2)
$$


a contradiction since for $x=1$ we have

$$
1+x \equiv 0 \quad(\bmod 2)
$$

and

$$
\sum_{j=0}^{m-1} x^{j 2^{\nu}} \equiv m \equiv 1(\bmod 2)
$$

One can generalize the problem considered here in the following way. Let $X$ be a compact topological space, $f: X \rightarrow X$ a continuous map and $C(X)$ the space of all continuous real functions on $X$ with the topology of uniform convergence. We can define a continuous map $T: C(X) \rightarrow C(X)$ by the formula

$$
\left.(T \varphi)(x)=\mid \varphi(x)-\varphi_{(}^{\prime} f(x)\right) \mid .
$$

Then the problem is again to investigate the asymptotic behaviour of the trajectories of the map $T$. We can also consider other types of convergence, not necessarily uniform.

The case investigated here is obtained from this general problem by taking $X$ consisting of $n$ points and $f$ - a cyclic permutation of $X$. Another interesting case can be obtained by taking $X=S^{1}$ (a circle) and $f$ an irrational rotation (i.e. a rotation by an angle non-commensurable with $\pi$ ). Then the conjecture of M. Skalba (unpublished) says that all trajectories converge to 0 . 


\section{REFERENCES}

[1 ] L. Carlitz and R. Scoville, Solution of Problem 69-1, SIAM Review 12 (1970), 298-300.

[2] B. Freeman, The four numbers game, Scripta Math. 14 (1948), 35-47.

[3 ] M. Lotan, A problem in difference sets, Amer. Math. Monthly 56 (1949), 535-541.

[4] Z. Nitecki, Differentiable dynamics, MIT Press, Cambridge, Massachusetts, 1971.

\section{ADDRESSES OF THE AUTHORS}

M. MISIUREWICZ

INSTITUTE OF MATHEMATICS

WARSAW UNIVERSITY

PKiN IX p.

00-901 WARSZAWA

POLAND

A. SCHINZEL

MATH. INSTITUTE P.A.N.

P.O. BOX 137, 00-950, WARSZAWA

POLAND

(RECEIVED BY EDITORS ON TTH FEBRUARY 1989) 
Note added on April 12. Here are further references for the case of integers.

E.R. Berlekamp, The design of slowly shrinking labelled squares, Math. Comp. 29 (1975), 25-27.

M. Burmester, R. Forcade, E. Jacobs, Circles of numbers, Glasgow Math. J. 19 (1978), 115-119.

C. Ciamberlini and A. Marengoni, Su una interessante curiosità numerica, Periodico Math. (4) 17 (1937), 25-30.

A. Ludington Furno, Cycles of differences of integers, J. Number Theory $13(1981), 255-261$.

R. Miller, A game with $n$ numbers, Arner. Math. Monthly 85 (1978), 183-185.

P. Zvengrowski, Iterated absolute differences, Math. Mag. 52 (1979), 36-40. 